\title{
The Effects and Mechanisms of the Rapamycin-eluting Stent in Urethral Stricture Prevention in Rabbits
}

\author{
Teng Zhang ${ }^{1,2}{ }^{\oplus}$, Wei Zhao ${ }^{1} \oplus$, Tengzhou Ren ${ }^{1}$, Jie Chen ${ }^{1} \oplus$, Zhiwei Chen ${ }^{1} \oplus$, Yan Wang ${ }^{1}$, \\ Xiaoju Cheng ${ }^{1}$, Jie $\mathrm{Wu}^{1} \oplus$, Chaoyong Yuan ${ }^{1}$, Tao $\mathrm{He}^{1} \odot$
}

${ }^{1}$ Third Affiliated Hospital of Zunyi Medical University (First people's Hospital of Zhuyi), Zhuyi, China

${ }^{2}$ First people's Hospital of Guiyang, Guiyang, China

Background: Rapamycin was shown to reduce transforming growth factor $\beta 1$ (TGF- $\beta 1$ ) expression, inhibit the Mammalian target of rapamycin function, and prevent TGF- $\beta 1$-induced pulmonary fibrosis. Rapamycin-eluting stents (RES) were successfully used to prevent coronary artery restenosis. Urethral stricture is one of the most challenging problems in urology. Thus, combining the pharmacological effects of rapamycin and the mechanical support of the stent on the urethra may prevent urethral stricture formation. However, the use of RES for urethral stricture treatment has not been studied.

Aims: To observe the effects of RES in urethral stricture in a rabbit model.

Study Design: Animal experimentation.

Methods: Twenty adult male New Zealand rabbits were randomly divided into control, urethral stricture model, bare-metal stent, and RES groups. The rabbits in the control group underwent urethroscopy alone without electrocoagulation. The rabbit model of urethral stricture was established by electrocoagulation using a self-made electrocoagulation device under direct vision using ureteroscopy. After model establishment, the rabbits in the bare-metal stent and RES groups received stent placement by ureteroscopy. On day 30, retrograde urethrography was performed to assess urethral stricture formation, ureteroscopy to remove the stents, and histological examinations to assess the degree of fibrosis. Reverse transcription-quantitative polymerase chain reaction (RT-qPCR) and western blot analysis were used to evaluate the expression levels of TGF- $\beta 1$, Smad3, and matrix metalloproteinase 1 (MMP1).

Results: Urethral stricture formation was seen in the model group, whereas not in the bare-metal stent group. The bare-metal stents did not displace but were difficult to remove. In the RES group, RES was dislodged in itself at postoperative day 27 in one rabbit, whereas successfully removed by ureteroscopy in the remaining four rabbits, and urethral stricture formation was not seen on retrograde urethrography after stent removal. Histological examination revealed a large number of dense fibroblasts and blue-stained collagen fibers in the bare-metal stent group, whereas the number of fibroblasts and collagen fibers under the mucosa was reduced in the RES group. RT-qPCR and Western blot analyses showed that the messenger ribonucleic acid (mRNA) and protein expression of TGF- $\beta 1$ and Smad3 was significantly decreased, and mRNA and protein expression of MMP1 was significantly increased in the RES group than that in the model $(P<0.001)$ and bare-metal stent groups $(P<0.001)$.

Conclusion: RES can effectively prevent electrocoagulation-induced urethral stricture in rabbits. The mechanism may be related to the effect of rapamycin on inhibiting TGF- $\beta 1$ and Smad 3 expression and promoting MMP1 expression in urethral tissues.

Corresponding author: Teng Zhang, Third Affiliated Hospital of Zunyi Medical University (First people's Hospital of Zhuyi), Zhuyi, China e-mail:wyly11@163.com

Received: April 15, 2021 Accepted: November 11, 2021 Available Online Date: March, 14, 2022 • DOI: 10.4274/balkanmedj.galenos.2021.2021-4-77

Available at www.balkanmedicaljournal.org

ORCID iDs of the authors: T.Z. 0000-0002-2391-5639; W.Z. 0000-0002-1624-4590; T.R. 0000-0002-6469-2988; J.C. 0000-0002-5817-7349; Z.C. 0000-0002-7431-5473; Y.W. 0000-0003-1044-5209; X.C. 0000-0001-8456-7300; J.W. 0000-0003-2770-1555; C.Y. 0000-0003-3032-747X; T.H. 0000-0001-5626-9402.

Cite this article as:

Zhang T, Zhao W, Ren T, Chen J, Chen Z, Wang Y, Cheng X, Wu J, Yuan Z, He T. The Effects and Mechanisms of the Rapamycin-eluting Stent in Urethral Stricture Prevention in Rabbits. Balkan Med J.; 2022; 39(2):107-14.

Copyright@Author(s) -Available online at http://balkanmedicaljournal.org/ 


\section{INTRODUCTION}

Urethral stricture is one of the most challenging problems in urology, which is highly recurrent and is defined as the narrowing of luminal urethral diameter caused by extensive fibrosis of the urethral epithelial tissues and corpus spongiosum, hypertrophic scar formation, and contraction. With progressive urethral lumen narrowing, varying degrees of obstructive symptoms may occur. ${ }^{1}$ Urethral stricture is most often secondary to urethral trauma and iatrogenic injury, with a gradually increasing number of cases. ${ }^{2}$ Treatment options include urethral dilation, direct visual internal urethrotomy, and urethroplasty. ${ }^{3}$ The choice of treatment depends on the location and length of the stricture and surgeon experience. Even with the most appropriate treatment option, several complications can arise. Stricture recurrence remains a common complication, which can make subsequent treatment more difficult and lead to a poor prognosis. ${ }^{4}$ Therefore, preventing the recurrence of urethral stricture is particularly important. Some treatment methods have been reported; however, no effective method was found for preventing and treating urethral stricture recurrence.

Urethral stricture is caused by a fibrotic process that is initiated by urethral tissue damage repair, which is characterized by excessive fibroblast proliferation and increased extracellular matrix secretion (mainly collagen). Urethral stricture is formed when the urethral tissues are replaced by dense non-elastic collagen fibers. Fibroblasts are closely related to urethral stricture occurrence. ${ }^{5}$ Transforming growth factor $\beta 1$ (TGF- $\beta 1$ ) is a potent chemokine for fibroblasts and an important mediator of fibrosis, which induces the production and secretion of collagen in fibroblasts, thereby triggering tissue fibrotic disorders. ${ }^{6}$ Smad3 is a downstream signaling protein of TGF- $\beta 1$. TGF- $\beta 1$ binds to its receptors on the cell membrane, stimulates phosphorylation of $\mathrm{Smad} 2 / 3$, which can form a functional complex with Smad4, and move into the nucleus to activate the gene expression of encoding collagen. ${ }^{7}$ Additionally, matrix metalloproteinases (MMPs) degrade structural proteins of the extracellular matrix. MMP1 is involved in the degradation of types I and III collagen, thus increased MMP1 expression can inhibit scar tissue formation. ${ }^{8,9}$

The use of drugs on the above-mentioned targets to prevent and treat tissue fibrosis has become a research hotspot. Many researchers have turned their attention to scar tissue formation inhibition for urethral stricture prevention and treatment. Presently, rapamycineluting stents (RES) have been successfully used to prevent coronary artery restenosis. ${ }^{10}$ However, satisfactory results with its use in urethral stricture treatment have not yet been reported. Studies have shown that rapamycin can prevent organ fibrotic diseases such as liver, pulmonary, and renal fibrosis. ${ }^{11}$

By combining the pharmacological effects of rapamycin and the mechanical support of the stent on the urethra (tubular organ), RES placement into the urethra was assumed to sustain and slowly release rapamycin, resulting in a constant drug concentration that acts on the urethral tissues. Therefore, this study established a rabbit model of urethral stricture by urethral electrocoagulation, placed RES into the urethra of rabbits, and observed its effects in urethral stricture formation.

\section{MATERIALS AND METHODS}

\section{Experimental Animals}

A total of 20 adult male New Zealand rabbits, weighing $2.52 \pm 0.25$ kg were purchased from Chongqing Tengxin Biotechnology Co., Ltd., China (license number: SCXK [Chongqing] 2017-0010). The rabbits were housed one per cage, and the room temperature was kept at $15-26{ }^{\circ} \mathrm{C}$. They were regularly fed and had free access to water. The experiment was approved by the Committee for Animal Experimentation of Third Affiliated Hospital of Zunyi Medical University.

\section{Methods}

All rabbits were randomly divided into control, urethral stricture model, bare-metal stent, and RES groups, with 5 rabbits in each group. All rabbits were anesthetized by intraperitoneal injection of $10 \%$ chloral hydrate $(300 \mathrm{mg} / \mathrm{kg})$ and fixed in the supine position on the surgical table, the penis was fully exposed. In the control group, rabbits underwent ureteroscopy (Wolf F8/9.8, Richard Wolf GmbH, Knittlingen, Germany) to observe the urethra, but did not undergo urethral electrocoagulation. In the model group, 10-mm-long circumferential electrocoagulation of the urethra was performed using a self-made electrocoagulation guidewire device under direct vision with ureteroscopy, which reached deeper into the muscular layer and was performed $2.0 \mathrm{~cm}$ distal to the verumontanum. In the bare-metal stent group, a bare-metal stent (peripheral stent, $4 \times 18 \mathrm{~mm}$, Cordis, Shanghai, China) was placed at the site of electrocoagulation injury under direct vision with ureteroscopy after model establishment. Then the stents were released to cover the site of electrocoagulation injury after selfexpansion. Rapamycin-eluting cobalt-chromium stent (Firebird 2, $4 \times 18 \mathrm{~mm}$, rapamycin dosage $160 \mathrm{ug}$, Microport Shanghai, China) was placed in the same way as mentioned above (Figure 1).

All operations were performed by the same urologist. On postoperative day 30 , retrograde urethrography was performed to assess the presence and absence of urethral stricture formation, then the stents were removed by ureteroscopy. After the rabbits were sacrificed by air embolization, the urethral tissues were collected, stored in a refrigerator at $-80{ }^{\circ} \mathrm{C}$, and fixed in a $10 \%$ formalin solution for pathological evaluation.

\section{Pathological Examination}

Urethral tissue samples were fixed in 10\% formalin solution and embedded in paraffin. Then sections $(5 \mu \mathrm{m})$ were made and stained using hematoxylin and eosin (HE) and Masson staining, respectively. The degree of fibrosis was observed.

\section{Western Blot Analysis}

The total protein from the urethral tissues of the rabbits was extracted, and protein concentration was determined using the BCA protein assay kit. A total of $20 \mu \mathrm{g}$ of protein was loaded onto the SDS-PAGE, separated using electrophoresis, and electrotransferred onto a poly (vinylidene fluoride) membrane. Then, the membrane was blocked at room temperature for $60 \mathrm{~min}$, incubated with antibodies for TGF- $\beta 1$ (diluted at 1:1000, Abcam, 
UK), Smad3 (diluted at 1:1000, GeneTex, USA), MMP1 (diluted at 1:1000, Proteintech, Wuhan, China), and $\beta$-actin (diluted at $1: 1000$, Proteintech, Wuhan, China) at a constant temperature of 4 ${ }^{\circ} \mathrm{C}$ overnight. The membrane was then washed and incubated with a secondary antibody at room temperature for $60 \mathrm{~min}$. Afterward, the membrane was subjected to an enhanced chemiluminescence reagent. Reactions were detected by exposure on the gel imager, and images were obtained. The relative expression of proteins was quantified by ImageJ software. All experiments were repeated three times, independently.

\section{Reverse transcription-quantitative polymerase chain reaction (RT-qPCR)}

Total ribonucleic acid (RNA) was isolated from approximately 20 mg of urethral tissues using a total RNA extraction kit, and the RNA concentration was measured using a nucleic acid detector (Thermo Scientific ${ }^{\circledR}$ USA). Then the total RNA was reversetranscribed into complementary deoxyribonucleic acid using a reverse transcription kit (Takara Bio, Japan). RT-qPCR was carried out with the SYBR Green on a Real-time PCR System (Bio-Rad, USA). The primer sequences are shown in Table 1. Data were normalized to the mRNA levels of glyceraldehyde-3-phosphatehydrogenase (GAPDH) that was used as a housekeeping gene. Results were expressed as cycle threshold $(\mathrm{Ct})$ value, which is defined as the number of required cycles for the fluorescent signal that exceeds the background level. $\Delta \mathrm{Ct}$ value, i.e., the difference in $\mathrm{Ct}$ values for the target gene and GAPDH, were calculated. Relative gene expression was analyzed using the $2^{-\Delta \Delta C T}$ method. Three duplicate holes were made for each sample and all experiments were repeated three times, independently.
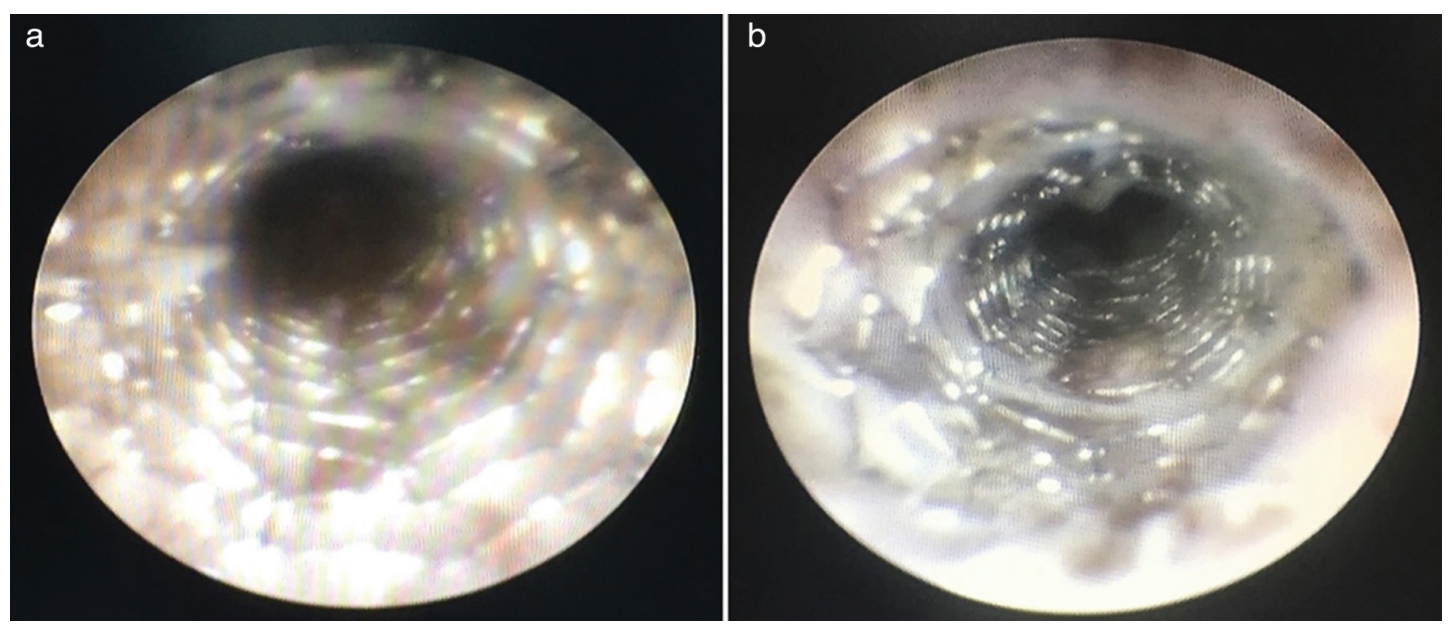

FIG. 1. Stent placement after establishing rabbit model of urethral stricture (a: rapamycin-eluting stent group; b: bare-metal stent group).

TABLE 1. PCR Primers Used in This Study

\begin{tabular}{lccc}
\hline Primers & GenBank Acc. & Forward sequence $\left(5^{\prime}{ }^{\prime} 3^{\prime}\right)$ & Reverse sequence $\left(5^{\prime}-3^{\prime}\right)$ \\
\hline TGF- $\beta 1$ & XM_008249704 & AACGATTCCTGGCGCTACCT & CCGGAACTGATCCCGTTGAT \\
Smad3 & XM_002718176 & ATGAGGTTTGCGTGAATCCCTAC & TGCTGTGGTTCATCTGGTGGTC \\
MMP1 & XM_001171139 & AGTCAGCCAGTAGGCCCACAGA & GTAGTAGGGATTTGCCCGCATG \\
GAPDH & XM_001082253 & GACCTGCCGCCTGGAGAAAG & CGACCTGGTCCTCGGTGTAG \\
\hline
\end{tabular}

In this experiment, the sample size was equal to 5 per group with an alpha error of 0.05 , a power of $>80 \%$, and an effect size of 0.9 . The Statistical Package for the Social Sciences 18.0 software was used to perform statistical analysis. The normality of continuous data was determined using the Kolmogorov-Smirnov test. Continuous variables with normal distribution were presented as mean \pm standard deviation and analyzed using the one-way analysis of variance followed by Tukey's honestly significant difference (HSD) test (assumption of homogeneity of variance) or Dunnett's T3 (nonassumption of homogeneity of variance) for pairwise comparisons. Analysis was performed with a significance level set at $\alpha=0.05 . P<0.05$ was considered statistically significant.

\section{RESULTS}

No deaths occurred in all four groups, as well as changes in mental status, food intake, behavioral activities, urination, and defecation. In the RES group, the stent was dislodged in itself at postoperative day 27 in one rabbit, whereas successfully removed by ureteroscopy in the remaining four rabbits. Stent removal was difficult in the bare-metal stent group.

\section{Results of Retrograde Urethrography}

No urethral stricture was observed in both the control, baremetal stent, and RES groups. Urethral stricture formation was observed in all five rabbits of the model group (Figure 2B), and postoperative measurement showed that the internal diameter of the urethral stricture segment was $2.51 \pm 0.25 \mathrm{~mm}$, and compared with the normal distal urethral segment, the percentage narrowing 
of the stricture segment ranged from $62.13 \%$ to $72.27 \%$, which were $>50 \%$, suggesting significant urethral narrowing. ${ }^{12}$

In the RES group, the stent lumen was unobstructed with a few necrotic flocculent attachments around it under observation by ureteroscopy. After stent removal, the urethral mucosa was seen to be pale, without stricture ring or damaged urethral mucosa, and with an unobstructed lumen (Figure 3).

\section{Hematoxylin and Eosin Staining}

A large number of dense fibroblasts under the mucosa were seen in the model group, which were significantly higher than those in the control group. A large number of dense fibroblasts were also seen in the bare-metal stent group, whereas the number of fibroblasts under the mucosa was reduced in the RES group (Figure 4).

\section{Masson Staining Results}

A large amount of blue-stained, dense collagen fibers under the urethral mucosa were visible in the model group, which were significantly higher than those in the control group. A large amount of blue-stained collagen fibers were also seen in the baremetal stent group, whereas significantly decreased blue-stained collagen fibers under the mucosa were observed in the RES group (Figure 5).
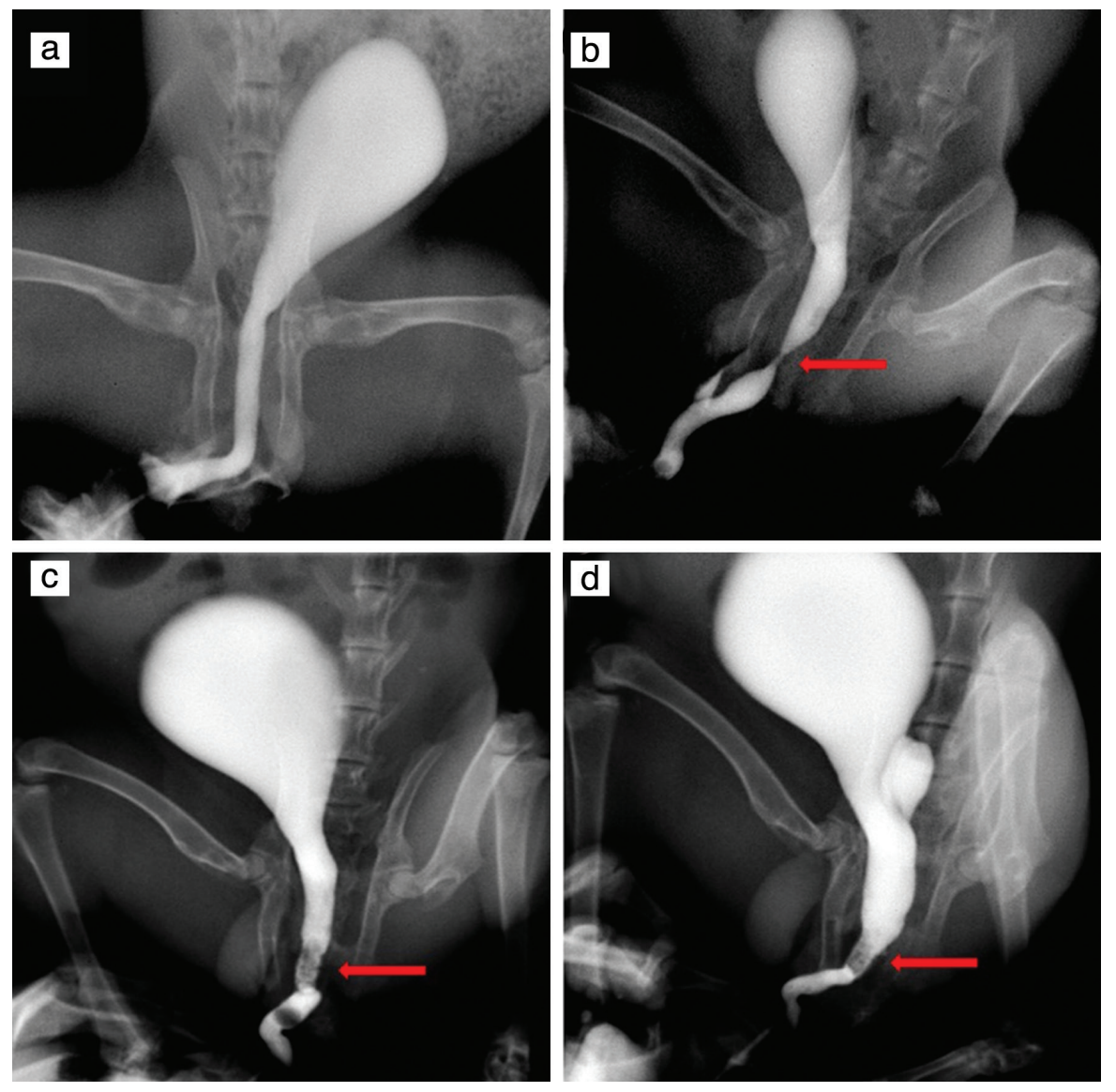

FIG. 2. Retrograde urethrography in rabbits of four groups. a: control group; b: model group; c: bare-metal stent group; d: rapamycin-eluting stent group. Arrows show urethral stricture and stent placement.

\section{RT-qPCR Results}

Statistically significant differences were found in the mRNA expression of TGF-b1 ( $\mathrm{F}=24.655, P<0.001), \operatorname{Smad} 3(\mathrm{~F}=21.874$, $P<0.001)$, and MMP1 (F $=44.752, P<0.001)$ among the four groups. Tukey's HSD test was used for pairwise comparison and revealed that the mRNA expression of TGF-b1, Smad3, and MMP1 in the model group was significantly higher than that in the control group $(P<0.001)$. The expression of TGF-b1 and Smad3 was significantly lower and MMP1 expression was significantly increased in the RES group than that in the model $(P<0.001)$ and bare-metal stent groups $(P<0.001)$. No significant difference was found in TGF-b1, Smad3, and MMP1 expression between the model group and bare-metal stent groups $(P>0.05$, Figure 6$)$.

\section{Western Blot Analysis Results}

Statistically significant differences were found in the protein levels of TGF-b1 ( $\mathrm{F}=20.317, P<0.001), \operatorname{Smad} 3(\mathrm{~F}=26.751, P<$ $0.001)$, and MMP1 ( $\mathrm{F}=32.651, P<0.001)$ among the four groups. Tukey's HSD test was used for pairwise comparison and revealed that the expression of TGF-b1, Smad3, and MMP1 in the model group was significantly increased than that in the control group $(P<0.001)$. The expression of TGF-b1 and Smad3 was significantly decreased, and MMP1 expression was significantly increased in the 
RES group than that in the model $(P<0.001)$ and bare-metal stent groups $(P<0.001)$. No significant difference was found between the model and bare-metal stent groups $(P>0.05$, Figure 7$)$.

\section{DISCUSSION}

Urethral stricture, especially recurrent urethral stricture, is a very difficult problem to treat. ${ }^{13}$ Urethral dilatation and direct visual internal urethrotomy are more commonly used to temporarily improve the urine flow rate; however, these improvements only last a short time, and reoperation is needed. Repeated dilation or internal urethrotomy may aggravate the formation of urethral scar tissues, which makes the subsequent treatment difficult. ${ }^{14}$ Urethroplasty is considered the gold-standard treatment of complex urethral stricture but is a more complex procedure, and many patients develop recurrence after surgery. ${ }^{15}$ If treatment for urethral stricture relies only on surgical treatment, the results are not satisfactory for patients and urologists. The use of drugs to inhibit urethral scar formation is an option to address the challenge in urethral stricture treatment but will not replace surgical treatment and can be used as an adjunct for surgery to improve the outcomes.

Presently, most scholars focus on rapamycin. Rapamycin is a macrolide antibiotic that is produced by Streptomyces hygroscopicus and is known to have immunosuppressive effects and anti-proliferative properties, which have been shown to inhibit the effects on fibrosis and are used in anti-cancer and anti-fibrotic therapies. ${ }^{16}$ Studies have found that rapamycin can reduce TGF- $\beta 1$
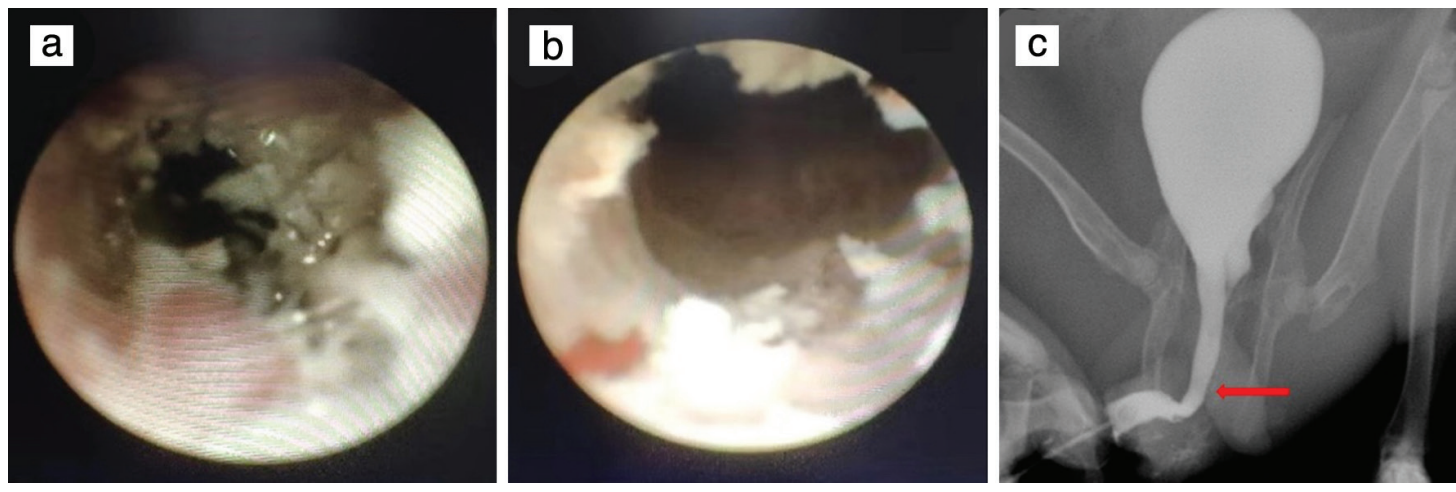

FIG. 3. Observation after stent removal in the rapamycin-eluting stent group (a: before stent removal, b: after stent removal, c: Images from urethrography).
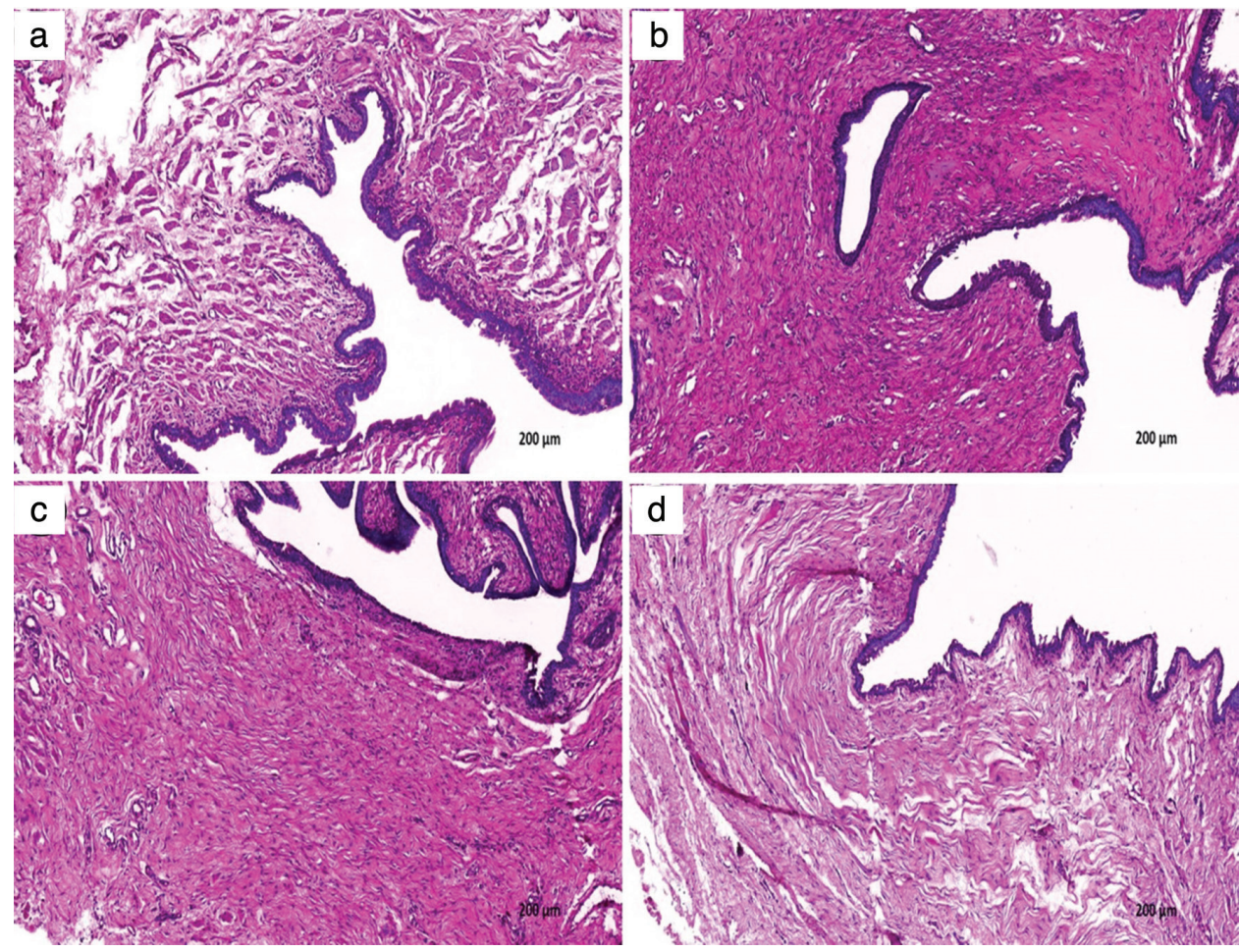

FIG. 4. The number of fibroblasts was obviously reduced in the RES group than model group and bare-metal stent group. Representative urethral stricture tissue sections of a control group, b model group, c bare-metal stent group, d RES group, following HE-staining (magnification $\times 100$ ). 


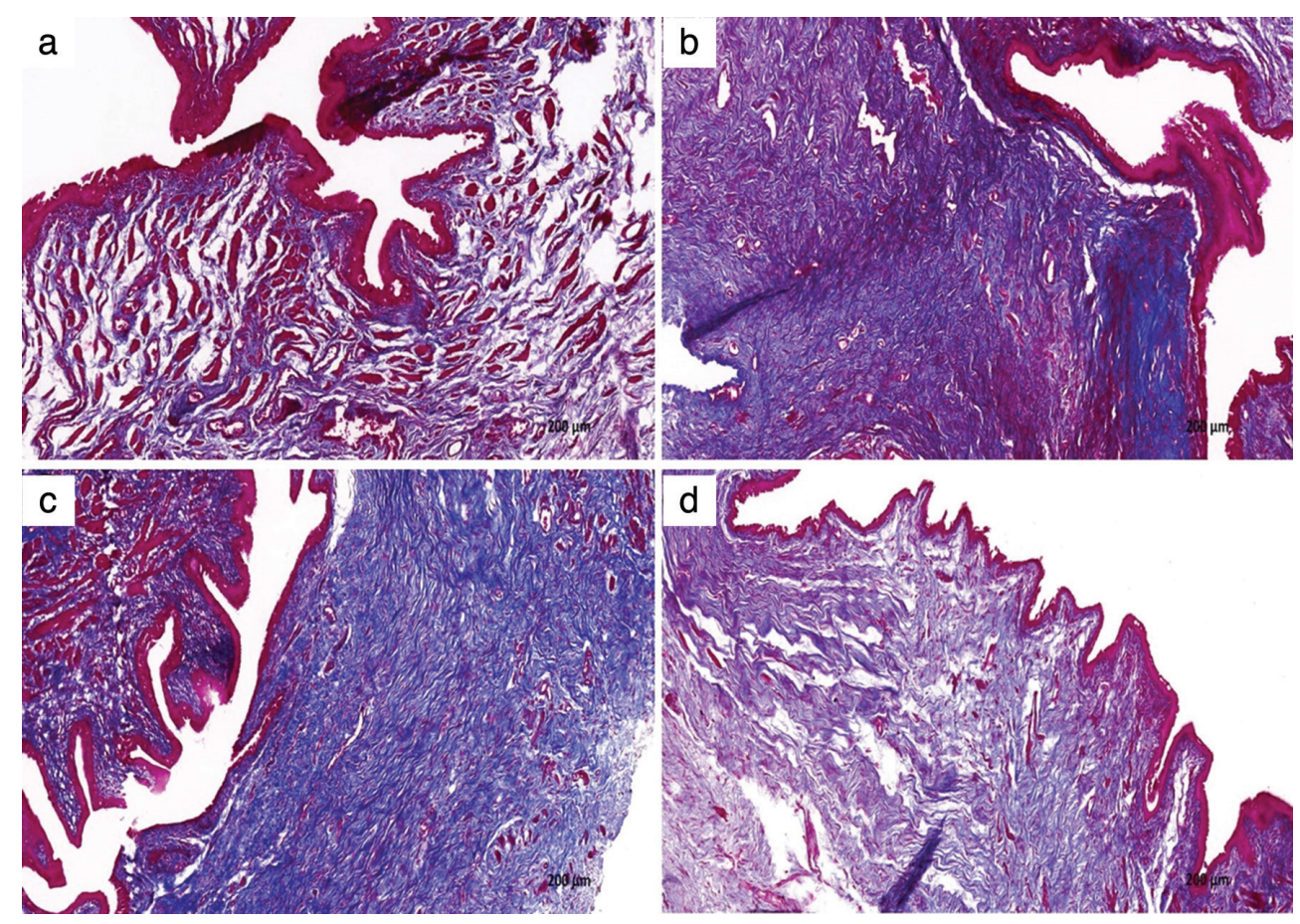

FIG. 5. The amount of blue-stained collagen fibers was obviously reduced in the RES group than model group and bare-metal stent group. Representative urethral stricture tissue sections of a control group, b model group, c bare-metal stent group, d RES group, following Masson-staining (magnification $\times 100$ ).
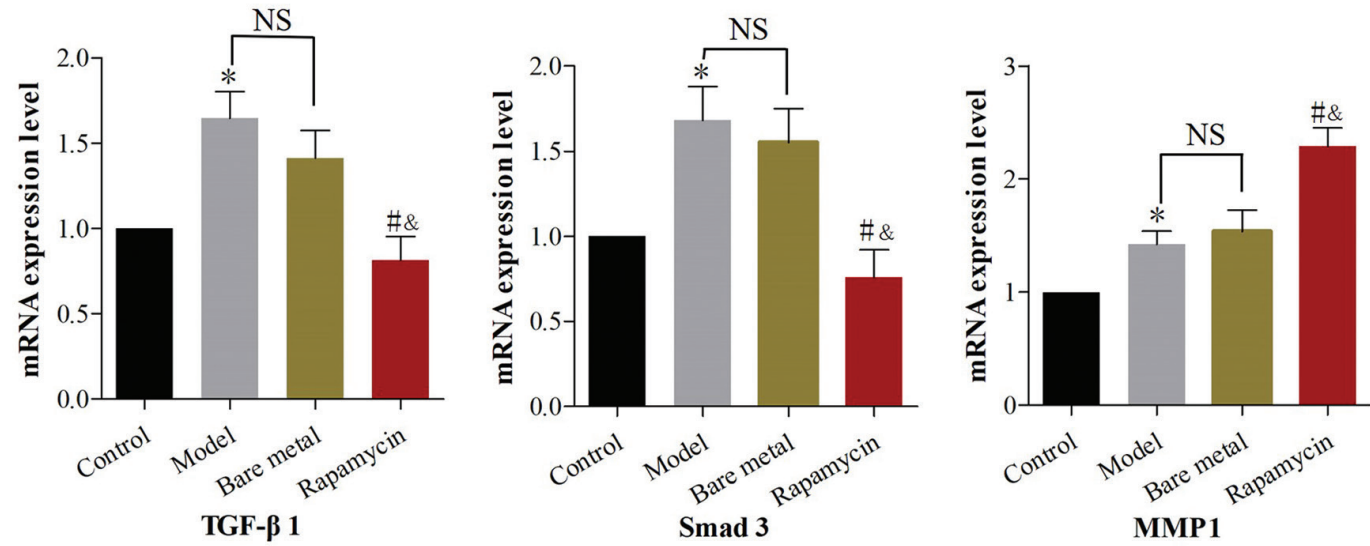

FIG. 6. mRNA expression of TGF-b1, Smad3, and MMP1 in each group. ${ }^{*} P<0.001$, vs control group; \#P<0.001, vs model group; \& $P<0.001$, vs bare-metal stent group; NS indicated no significant differences between the model and bare-metal stent groups.

expression in animal models of renal fibrosis ${ }^{17}$ and prevent TGF- $\beta 1$ induced pulmonary fibrosis ${ }^{18}$, but the mechanism of action remains unclear. Rapamycin can bind to FK-binding protein 12 and inhibit the function of the Mammalian target of rapamycin (mTOR), which is a key protein of mTOR complex 1 (mTORC1) that can inhibit the downstream signaling factors of the mTOR signaling pathway. ${ }^{19}$ Studies revealed crosstalk between the TGF- $\beta / \mathrm{Smad}$ and mTOR pathways, and TGF- $\beta$-induced collagen expression was affected by mTORC1 activity. ${ }^{20,21}$ Therefore, the inhibitory effect of rapamycin on TGF- $\beta 1$ and Smad3 was speculated to be mediated by the TGF- $\beta / \mathrm{Smad} / \mathrm{mTOR}$ signaling pathway, but this hypothesis needs further investigation. Chong et al. ${ }^{22}$ established a rabbit model of urethral stricture by urethral electrocoagulation. After treatment with rapamycin by retrograde urethral irrigation, they found that rapamycin can prevent urethral stricture. Huang et $\mathrm{al}^{23}$ found that rapamycin can promote MMP1 expression, reduce collagen fiber formation, and prevent urethral stricture. However, in their study ${ }^{23}$, rapamycin is administrated by urethral irrigation and, the drug concentration is easily diluted by urine and is excreted with urine, leading to a short drug action duration. An in vitro study conducted by Delai Fu et al. ${ }^{24}$ found that rapamycin can inhibit the growth of human urethral scar tissue and collagen expression although sustained action of the drug is maintained, this is an in vitro study. The application and placement of a rapamycin 

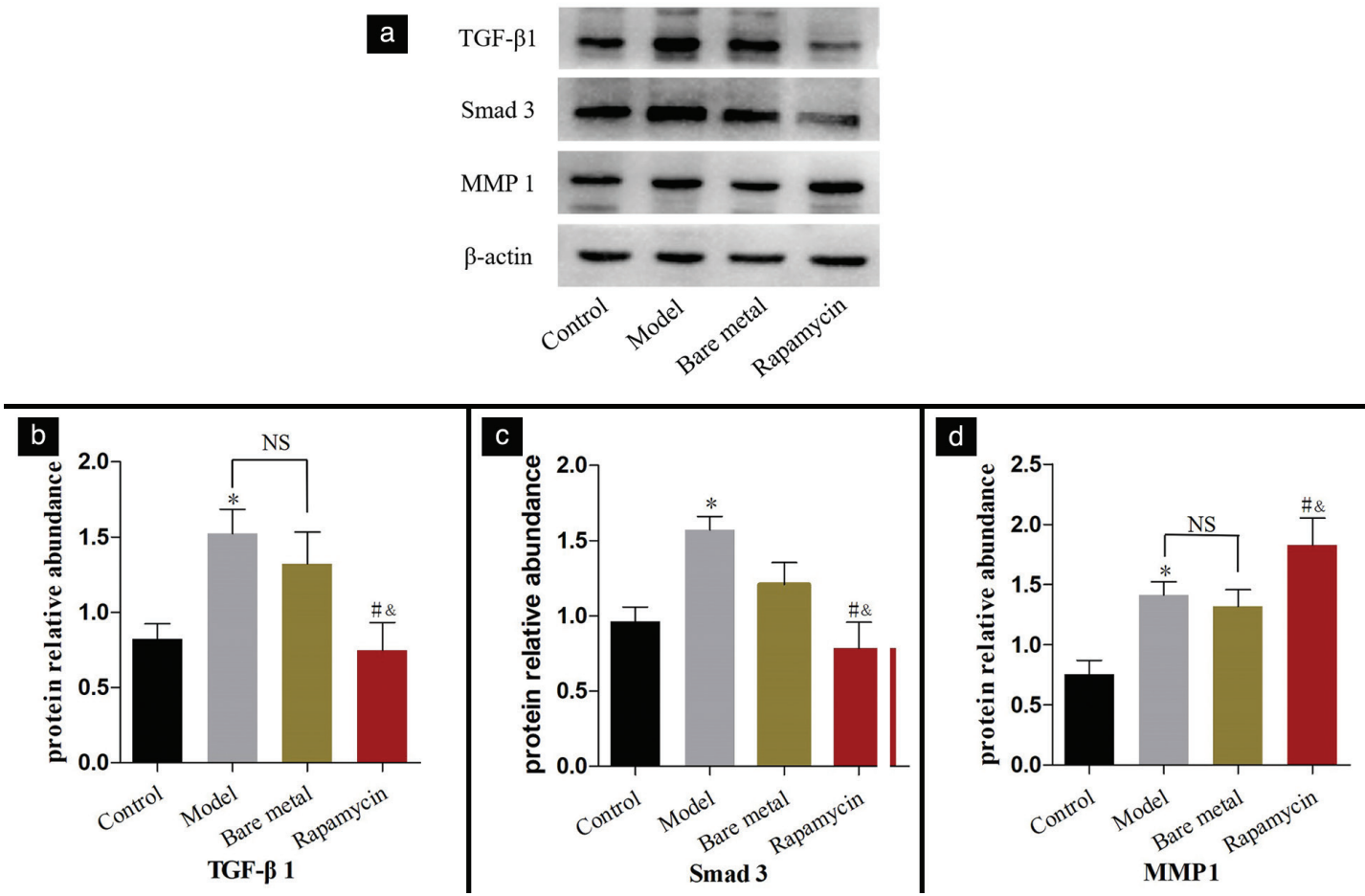

FIG. 7. a: protein bands corresponding to TGF-b1, Smad3, and MMP1 in each group; b, c, d: expression of TGF-b1, Smad3, and MMP1 in each group. ${ }^{*} P<0.001$, vs control group; $\# P<0.001$, vs model group; \& $P<0.001$, vs bare-metal stent group; NS indicated no significant differences between the model and bare-metal stent groups.

carrier into rabbits' urethra were assumed to sustain and slowly release the drug to act on the urethral tissues.

The innovation of this study is the first use of RES for urethral stricture prevention and treatment. In this study, after the rabbit's urethra was injured by electrocoagulation, RES was placed into the rabbits' urethra at the injury site under direct ureteroscopic vision, and the stent had well expanded (Figure 1). The animals did not exhibit behavioral changes, and no deaths occurred after the surgery, indicating that RES placement into the urethra is feasible. After stent placement, no urethral stricture was observed in rabbits of the bare-metal stent and RES groups. The degree of fibrosis was significantly reduced in the RES group compared with the model and bare-metal stent groups, indicating that sustained action of rapamycin alters the fibrotic microenvironment. After RES achieves the therapeutic effect, the stent can be removed without damaging the urethra, thereby avoiding the complications due to long-term stent placement. The urethral stricture was not observed in the bare-metal stent group, probably due to the short observation period in the present study.

Our findings revealed significantly reduced expression of TGF- $\beta 1$ and Smad 3 and increased MMP1 expression in the RES group compared with the model and bare-metal stent groups. However, no significant difference was found in the expression of TGF- $\beta 1$, Smad3, and MMP1 between the model and baremetal stent groups. The bare-metal stent is not coated with the drug group, thud fibrotic changes in the urethral tissues cannot be effectively inhibited. Urethral stricture treatment by bare-metal stent placement can keep the lumen open in the short term, but the long-term stimulating effect of bare-metal stents on urethral tissues can cause squamous metaplasia of urethral epithelium, fibrosis, and inflammatory hyperplasia, and hyperplastic granulation tissues can grow into the urethral lumen through the stent mesh, causing stent lumen obstruction and stent removal difficulty. ${ }^{25}$ The MMPs are regulated by TGF- $\beta 1$, and TGF- $\beta 1$ may down-regulate the expression of MMPs through the TGF- $\beta 1$ inhibitory element in the MMP promoters. ${ }^{26}$ Another study found that rapamycin can increase MMP1 expression, and proposed that rapamycin may cause the activation of the c-Jun N-terminal kinase cascade in dermal fibroblasts, resulting in an increased MMP1 gene promoter activity. ${ }^{27}$ The mechanism of action needs further investigation.

This study has some limitations, some released drugs can be excreted in the urine, and the dose of rapamycin on the stent in this study was $160 \mathrm{ug}$, thus further studies are needed to investigate whether this is the optimal drug dosage. Despite the limitations, our study demonstrates that rapamycin can inhibit urethral fibrosis and prevent urethral stricture, which may be used as a measure for the prevention of urethral stricture recurrence after surgery.

RES can prevent urethral strictures in rabbits, and the mechanism of action may be related to the effects of rapamycin on inhibiting TGF- $\beta 1$ and Smad3 expression and promoting MMP1 expression in urethral tissues. Drug-eluting stents exert potential value in urethral stricture prevention, which is worth further exploration. 
Ethics Committee Approval: The experiment was approved by the Committee for Animal Experimentation of Third Affiliated Hospital of Zunyi Medical University.

Data Sharing Statement:The data that support the findings of this study are available from the corresponding author upon reasonable request.

Author Contributions: Concept - Y.W., ; Design - Y.W., ; Supervision - Y.W., C.Y., T.H.; Data Collection and Processing - T.Z., W.Z., T.R., J.C., Z.C., X.C., J.W., C.Y., T.H.; Analysis and/or Interpretation - T.Z., W.Z., T.R., J.C., Z.C., X.C., J.W., C.Y., T.H.; Writing - T.Z.

Conflict of Interest: No conflict of interest was declared by the authors.

Funding: The study was supported by National Natural Science Foundation of China (No. $81660121)$.

\section{REFERENCES}

1. King C, Rourke KF. Urethral Stricture is Frequently a Morbid Condition: Incidence and Factors Associated With Complications Related to Urethral Stricture. Urology. 2019;132:189-194. [CrossRef]

2. Gottipamula S, Sundarrajan S, Chokalingam K, Sridhar KN. The effect of human amniotic epithelial cells on urethral stricture fibroblasts. J Clin Transl Res. 2019;5:4449. [CrossRef]

3. Verla W, Oosterlinck W, Spinoit AF, Waterloos M. A Comprehensive Review Emphasizing Anatomy, Etiology, Diagnosis, and Treatment of Male Urethral Stricture Disease. Biomed Res Int. 2019;2019:9046430. [CrossRef]

4. Heyns CF, van der Merwe J, Basson J, van der Merwe A. Treatment of male urethral strictures - possible reasons for the use of repeated dilatation or internal urethrotomy rather than urethroplasty. S Afr J Surg. 2012;50:82-87. [CrossRef]

5. Prihadi JC, Sugandi S, Siregar NC, Soejono G, Harahap A. Imbalance in extracellular matrix degradation in urethral stricture. Res Rep Urol. 2018;10:227-232. [CrossRef]

6. Lodyga M, Hinz B. TGF- $\beta 1$ - A truly transforming growth factor in fibrosis and immunity. Semin Cell Dev Biol. 2020;101:123-139. [CrossRef]

7. Fang $\mathrm{X}, \mathrm{Hu} \mathrm{X}$, Zheng $\mathrm{Z}$, et al. Smad interacting protein 1 influences transforming growth factor- $\beta 1 /$ Smad signaling in extracellular matrix protein production and hypertrophic scar formation. J Mol Histol. 2019;50:503-514. [CrossRef]

8. Chuang HM, Chen YS, Harn HJ. The Versatile Role of Matrix Metalloproteinase for the Diverse Results of Fibrosis Treatment. Molecules. 2019;24:4188. [CrossRef]

9. Siregar S, Farenia R, Sugandi S, Roesli RM. Effect of angiotensin II receptor blocker on TGF- $\beta 1$, MMP-1, and collagen type I and type III concentration in New Zealand rabbit urethral stricture model. Res Rep Urol. 2018;10:127-133. [CrossRef]

10. Zhu P, Zhou X, Zhang C, Li H, Zhang Z, Song Z. Safety and efficacy of ultrathin strut biodegradable polymer sirolimus-eluting stent versus durable polymer drug-eluting stents: a meta-analysis of randomized trials. BMC Cardiovasc Disord. 2018;18:170. [CrossRef]
11. Yanaba K. Strategy for treatment of fibrosis in systemic sclerosis: Present and future. J Dermatol. 2016;43:46-55. [CrossRef]

12. Meria P, Anidjar M, Brouland JP, et al. An experimental model of bulbar urethral stricture in rabbits using endoscopic radiofrequency coagulation. Urology. 1999;53:1054-1057. [CrossRef]

13. Zaid UB, Lavien G, Peterson AC. Management of the Recurrent Male Urethral Stricture. Curr Urol Rep. 2016;17:33. [CrossRef]

14. Horiguchi A, Shinchi M, Masunaga A, Ito K, Asano T, Azuma R. Do Transurethral Treatments Increase the Complexity of Urethral Strictures? J Urol. 2018;199:508514. [CrossRef]

15. Han JS, Liu J, Hofer MD, et al. Risk of urethral stricture recurrence increases over time after urethroplasty. Int J Urol. 2015;22:695-699. [CrossRef]

16. Arriola Apelo SI, Lamming DW. Rapamycin: An InhibiTOR of Aging Emerges from the Soil of Easter Island. J Gerontol A Biol Sci Med Sci. 2016;71:841-849. [CrossRef]

17. Liu CF, Liu H, Fang Y, Jiang SH, Zhu JM, Ding XQ. Rapamycin reduces renal hypoxia, interstitial inflammation and fibrosis in a rat model of unilateral ureteral obstruction. Clin Invest Med. 2014;37:E142. [CrossRef]

18. Saxton RA, Sabatini DM. mTOR Signaling in Growth, Metabolism, and Disease. Cell. 2017;168:960-976. [CrossRef]

19. Gao Y, Xu X, Ding K, Liang Y, Jiang D, Dai H. Rapamycin inhibits transforming growth factor $\beta 1$-induced fibrogenesis in primary human lung fibroblasts. Yonsei Med J. 2013;54:437-444. [CrossRef]

20. Rozen-Zvi B, Hayashida T, Hubchak SC, Hanna C, Platanias LC, Schnaper HW. TGF- $\beta /$ Smad3 activates mammalian target of rapamycin complex-1 to promote collagen production by increasing HIF-1 $\alpha$ expression. Am J Physiol Renal Physiol. 2013;305:F485-E494. [CrossRef]

21. Das F, Bera A, Ghosh-Choudhury N, Abboud HE, Kasinath BS, Choudhury GG. TGF $\beta$-induced deptor suppression recruits $\mathrm{mTORC} 1$ and not mTORC2 to enhance collagen I ( $\alpha 2)$ gene expression. PLoS One. 2014;9:e109608. [CrossRef]

22. Chong T, Fu DL, Li HC, et al. Rapamycin inhibits formation of urethral stricture in rabbits. J Pharmacol Exp Ther. 2011;338:47-52. [CrossRef]

23. Huang SL, Fu DL, Li HC, Zhang P, Chong T. The effect of rapamycin on TGFBI and MMP1 expression in a rabbit model of urethral stricture. Int Urol Nephrol. 2016;48:717-723. [CrossRef]

24. Fu D, Yin J, Huang S, Li H, Li Z, Chong T. Rapamycin Inhibits the Growth and Collagen Production of Fibroblasts Derived from Human Urethral Scar Tissue. Biomed Res Int. 2018;2018:7851327. [CrossRef]

25. De Vocht TF, van Venrooij GE, Boon TA. Self-expanding stent insertion for urethral strictures: a 10-year follow-up. BJU Int. 2003;91:627-630. [CrossRef]

26. Cui $\mathrm{N}, \mathrm{Hu} \mathrm{M}$, Khalil RA. Biochemical and Biological Attributes of Matrix Metalloproteinases. Prog Mol Biol Transl Sci. 2017;147:1-73. [CrossRef]

27. Tamaki Z, Asano Y, Kubo M, et al. Effects of the immunosuppressant rapamycin on the expression of human $\alpha 2$ (I) collagen and matrix metalloproteinase 1 genes in scleroderma dermal fibroblasts. J Dermatol Sci. 2014;74:251-259. [CrossRef] 